\title{
Pharmacogenomics in type II diabetes mellitus management: Steps toward personalized medicine
}

This article was published in the following Dove Press journal:

Pharmacogenomics and Personalized Medicine

10 September 2009

Number of times this article has been viewed

\section{Peter Avery \\ Shaymaa S Mousa \\ Shaker A Mousa \\ The Pharmaceutical Research Institute, Albany College of Pharmacy and Health Sciences, Albany, NY, USA}

Correspondence: Shaker A Mousa Executive VP and Chairman of the Pharmaceutical Research Institute, Albany College of Pharmacy, One Discovery Drive, Rensselaer, NY 12 | 44, USA

$\mathrm{Tel}+\mathrm{I} 5186947397$

Fax +I 5186947567

Email shaker.mousa@acphs.edu

\begin{abstract}
Advances in genotype technology in the last decade have put the pharmacogenomics revolution at the forefront of future medicine in clinical practice. Discovery of novel gene variations in drug transporters, drug targets, effector proteins and metabolizing enzymes in the form of single-nucleotide polymorphisms (SNPs) continue to provide insight into the biological phenomena that govern drug efficacy and toxicity. To date, novel gene discoveries extracted from genome-wide association scans and candidate gene studies in at least four antidiabetic drug classes have helped illuminate possible causes of interindividual variability in response. Inadequate protocol guidelines for pharmacogenomics studies often leads to poorly designed studies, making it hard to formulate a definitive conclusion regarding the clinical relevance of the information at hand. These issues, along with the ethical, social, political, legislative, technological, and economic challenges associated with pharmacogenomics have only delayed its entry to mainstream clinical practice. On the other hand, these issues are being actively pursued and rapid progress is being made in each area which assures the possibility of gaining widespread acceptance in clinical practice.
\end{abstract}

Keywords: pharmacogenomics, genetics, pharmacokinetics, pharmacodynamics, personalized medicine, type 2 diabetes, pharmacotherapy, antidiabetic drugs, efficacy, and safety

\section{Introduction}

In the previous decade a major component of discussions involving disease prevention, therapy and prognosis has shifted from genetics to genomics. ${ }^{1-3}$ The completion of the Human Genome Project in 2003 and advances in genotyping technology have enabled researchers to explore the entire genome, updating their approach from looking at just genes to the genome as a whole, in what are referred to as genome-wide association studies (GWAS). ${ }^{4}$ Novel information regarding individual disease susceptibility, response to therapy and progression path has emerged from this approach at a relatively rapid pace, especially in the past two years. ${ }^{4,5}$ This is because a determinant of an individual's disease susceptibility, response to therapy, and progression are attributed, at least partly, to common variations in certain genes; mainly in the form of single nucleotide polymorphisms (SNPs). ${ }^{5}$ This approach has infiltrated many analytical fields to produce many hybridized genomic fields, which include toxicogenomics, proteomics, and pharmacogenomics. ${ }^{1,3}$ However, the focus here will be on how polymorphisms in an individual's genome can alter a response to drug therapy, an area referred to as pharmacogenomics.

Pharmacogenomics is an approach that has evolved from pharmacogenetics and become a new scope for the pharmaceutical and biomedical fields. ${ }^{1,3}$ Pharmacogenomics

submit your manuscript | www.dovepress.com 
promises a new drug selection process, which takes into account variations in an individual's genetic makeup to optimize pharmacokinetics and pharmacodynamics to ultimately increase drug efficacy and safety profile. ${ }^{6}$ In other words, creating genetically tailored drug regimens to optimize an individual's response. ${ }^{1,6}$ It is estimated that about $95 \%$ of the variability in drug response is due to genetic differences; accounting for these differences then would be highly beneficial, not only for the health care industry, but for patients themselves, decreasing the burden of treatment failures and adverse events on society. ${ }^{7,8}$

The pharmacogenomics approach has already supplied researchers with candidate genes and their translational ramifications on drug response in many complex disease states. $^{2}$ A popular focus of several GWAS and candidate gene analysis has been dedicated to understanding the implications of genetic variation in individuals diagnosed with type 2 diabetes mellitus (T2D). ${ }^{9}$ In recent years, a great deal of progress has been made in the form of establishing a genetic explanation for etiological mechanisms by which T2D develops and interindividual variability in response to standard therapy. ${ }^{10}$

According to 2007 surveys, the prevalence of diabetes is estimated at 246 million, which is roughly a $63 \%$ increase since the turn of the 21 st century. Perhaps more alarming is of the prediction of an increased prevalence in years to come, with an estimate of 380 million cases in the year $2025 .{ }^{11}$ In the United States alone, the prevalence of diagnosed and undiagnosed diabetes cases was 7.8\% (23.6 million) of the population, $90 \%$ to $95 \%$ of which are T2D. ${ }^{11}$ Additionally, DM is the leading cause of blindness, end-stage renal failure and lower extremity amputations, and increases the risk for many other life-threatening conditions such as heart disease and stroke by two to four times that of a nondiabetic. ${ }^{12}$ Financially speaking, an estimated one out of every five health care dollars are spent on diabetic care in the United States and about 174 billion total health care dollars spent annually. ${ }^{13}$

These numbers can be partly attributed to an aging population, unhealthy diet, increasing rates of obesity and sedentary lifestyles. ${ }^{11}$ Although obesity rates are increasing among all age groups, a significant contributor to these overwhelming prevalence trends is the increase in childhood obesity rates in developing countries. ${ }^{11,14}$ Therefore, it comes as no surprise that behavioral modifications (diet and exercise) are the initial recommendation for managing T2D. ${ }^{15,16}$ But when euglycemia cannot be achieved through dietary and exercise modification alone, supplementation with oral hypoglycemics and insulin, in some cases, is recommended. ${ }^{16}$ Type 2 DM is treatable with ten drug classes: biguanides, sulfonylureas (SUs), thiazolidinediones (TZD), meglitinides, $\alpha$-glucosidase inhibitors, amylin mimetics, glucagon-like peptide-1 (GLP-1) mimetics, dipeptidyl peptidase-4 (DPP-4) inhibitors, and insulin. ${ }^{16,17}$ However, there is a significant amount of variability in responses to these antidiabetic agents. Recent pharmacogenomic studies have linked antidiabetic drug response variability to genetic polymorphisms in transporters, targets, effector proteins and metabolizing enzymes. The purpose of this article is to explain how pharmacogenomics aims to achieve the best therapeutic outcome based on genomic insights, to ultimately lead to the development of "personalized medicine". ?

\section{Biguanide class (Metformin) Metformin's place in therapy and mechanism of action}

Introduced to the United States market in 1995, metformin has become a first-line therapy for the management of T2D and is widely prescribed in the United States. ${ }^{17}$ By 2006, metformin accounted for $37 \%$ of noninsulin prescriptions in the United States alone. ${ }^{17}$ Although the full pharmacological action profile remains unclear at this point, evidence suggests that the major action of metformin is exerted in the liver, primarily from the activation of adenosine monophosphate-activated protein kinase (AMPK). Activation (phosphorylation) of AMPK is done by serine-threonine kinase (LKB1), which suppresses hepatic gluconeogenesis, thereby, reducing glucagon-mediated glucose output by the liver. ${ }^{17,18}$ Metformin is able to exert glucose-lowering actions with a low risk of hypoglycemia, as well as reduce the likelihood of developing macrovascular (hypertension, atherosclerosis) and microvascular (neuropathy, retinopathy, nephropathy) complications, these qualities are what make it an attractive first-line therapy. ${ }^{17-19}$ However, metformin is not without adverse events such as diarrhea and nausea that occur in about $30 \%$ of patients; or a more serious but very rare side effect, lactic acidosis. ${ }^{17,18}$ Despite an exceptional efficacy and safety profile, several T2Ds (about 38\%) still fail to reach glycemic goals in metformin therapy. ${ }^{17}$

Recent studies suggest that interpatient variability in response to metformin therapy could be related to polymorphisms in the organic cation transporter (OCT) genes and/or the multidrug and toxin extrusion (MATE) genes (Table 1). ${ }^{17,18,20-22}$ The SLC22A1 and SLC22A2 genes encode for OCT1 and OCT2, respectively. ${ }^{22,23}$ OCT1 is primarily 
expressed in hepatocytes and enterocytes and mediate the uptake of metformin in these cells. ${ }^{17,24}$ Expressed predominately in the renal distal tubule, OCT2 functions to facilitate urinary elimination many xenobiotics, including metformin. ${ }^{18}$ The SLC47A1 and SLC47A2 genes encode for the MATE1 and MATE2-K proteins, respectively. ${ }^{25}$ Originally, the MATE proteins were found in bacteria and function to sustain cell equilibrium by excreting metabolic wastes and xenobiotics, but recently they have been shown to play a similar role in humans. ${ }^{26}$ The MATE1 protein is expressed in the bile canalicular membrane of hepatocytes and in the renal epithelium, whereas MATE2-K is expressed only in the renal epithelium. ${ }^{25}$ It has been demonstrated that metformin is an exceptional substrate for MATE1 and MATE2-K and that these proteins play a role in the elimination of metformin into the bile (MATE1) and urine (MATE1 and MATE2-K). ${ }^{25,27}$

\section{Role of SLC22AI polymorphisms in metformin response}

Wang and colleagues observed a 30-fold decrease in hepatic uptake and a 3- to 7-fold decrease in intestinal (duodenum, jejunum, ileum) uptake of metformin in $\mathrm{Oct}^{-/-}$mice compared to $O c t 1^{+/+}$mice. ${ }^{28}$ However, the renal distribution and elimination of metformin was found to be nearly equal when the two mice groups were compared. ${ }^{28}$ These results demonstrate that OCT1 is involved in hepatocyte and enterocyte uptake and that kidney uptake relied minimally, if at all, on OCT1 $1{ }^{28}$ Since kidney uptake was not affected by the presence or absence of OCT1, the authors concluded that distribution to the kidney was achieved primarily by another transport mechanism. ${ }^{28}$ The major findings of this study were that hepatic and intestinal uptake was accomplished through OCT1, but whether OCT1 had any influence on metformin's response or toxicity (ie, nausea, diarrhea, lactic acidosis) remained in question. ${ }^{28}$

In 2007, Shu and colleagues sought to discover whether OCT1 plays a role in the actions of metformin and the associated implications on drug response. ${ }^{21}$ Therefore, investigators initially looked at whether AMPK activation was associated with OCT1 expression. ${ }^{21}$ In order to evaluate this, metformin-stimulated AMPK activation of cells transfected with human OCT1 (HEK-OCT1 cells) were compared to control cells (empty vector-transfected cells). ${ }^{21}$ Metformin accumulated considerably more in the HEK-OCT1 cells, which also corresponded with a higher AMPK activation than that of the empty vector cells. ${ }^{21}$ This demonstrates that OCT1 is needed for metformin uptake and pharmacological actions in the liver in vitro. Next, investigators compared the in vivo uptake of metformin in $O c t 1^{-1-}$ mice with cells containing a functional Oct1 allele $\left(O c t 1^{+/+}\right.$and $\left.O c t 1^{+/-}\right)$ after a single oral dose of metformin. ${ }^{21}$ The results from this experiment were consistent with Wang and colleagues and other research that showed the hepatic uptake in wildtype mice $\left(\mathrm{Octl}^{+/+}\right.$and $\mathrm{Oct} \mathrm{1}^{+/-}$) was significantly (4.2-fold) higher in comparison to the $\operatorname{Oct}^{-/-}$mice. ${ }^{21,28}$ Additionally, metformin suppressed glucagon-stimulated glucose production in the wild-type mice, but not in the $\operatorname{Oct}^{-/-}$mice. ${ }^{21}$ Subsequently, the $\mathrm{Oct1}^{-/-}$and wild-type mice were fed the same high-fat diet for eight weeks to increase fasting plasma glucose (FPG) levels and were subjected to a five-day treatment with either saline or metformin. ${ }^{21}$ Wild-type mice receiving metformin therapy illustrated a reduction in FPG by over $30 \%$, but $\mathrm{Oct}^{-/-}$mice that received metformin did not have a notable reduction in FPG levels. ${ }^{21}$ In summary, Shu and colleagues revealed that metformin accumulation and AMPK activation are dependent on functional

Table I Possible role and effect of variations on metformin therapy

\begin{tabular}{|c|c|c|c|c|}
\hline & SLC22A I & SLC22A2 & SLC47A I & SLC47A2 \\
\hline Codes & OCTI & ОСТ2 & MATEI & MATE2-K \\
\hline Site & Hepatocytes, enterocytes & Renal distal tubule & $\begin{array}{l}\text { Bile canalicular membrane of } \\
\text { hepatocytes and in renal epithelium }\end{array}$ & Renal epithelium \\
\hline Action & $\begin{array}{l}\text { Mediates metformin uptake, } \\
\text { accumulation and pharmacological } \\
\text { action in the liver (AMPK activation) }\end{array}$ & $\begin{array}{l}\text { Facilitates urinary } \\
\text { elimination of metformin }\end{array}$ & Metformin secretion in bile and urine & $\begin{array}{l}\text { Metformin excretion } \\
\text { in urine }\end{array}$ \\
\hline Variants & $\begin{array}{l}\text { Decreased hepatic and intestinal } \\
\text { metformin uptake and accumulation. } \\
\text { Decreased AMPK activation. } \\
\text { Increased plasma glucose levels and } \\
\text { lower insulin levels }\end{array}$ & $\begin{array}{l}\text { Decreased metformin } \\
\text { clearance and increased } \\
\text { plasma concentration }\end{array}$ & $\begin{array}{l}\text { May affect glucose lowering effects of } \\
\text { metformin. With each } \mathrm{A}_{\text {allele there is }} \\
\text { an associated } 0.3 \% \mathrm{HbA}_{\mathrm{IC}} \text { reduction. } \\
\mathrm{G} \text { allele has no association with } \mathrm{HbA}_{\mathrm{IC}} \\
\text { reduction }\end{array}$ & \\
\hline
\end{tabular}


OCT1 expression and that the level of AMPK activation corresponded to suppression in glucagon-stimulated glucose production and reduced FPG levels. ${ }^{21}$ The final assessment was to determine whether OCT1 polymorphisms altered metformin response in humans by measuring metformin uptake in 12 known OCT1 variants (S14F, R61C, F160L, S189L, G220V, P341L, R342H, G401S, V408M, 420del, G465R, R488M) and comparing them to an OCT1-reference using HEK293 cells. This resulted in the identification of seven reduced function OCT1 variants (S14F, R61C, S189L, G220V, G401S, 420del, G465R; p < 0.001) in terms of metformin uptake and AMPK activation in transfected HEK293 cells. Next, humans expressing these genotypes were studied by measuring plasma glucose levels using a three-hour oral glucose tolerance test (OGTT), after receiving oral glucose and metformin treatment. The results showed that metformin in reference alleles produced a significant reduction in plasma glucose levels relative to the reduced function alleles. In addition, reference alleles were associated with significantly $(\mathrm{p}<0.05)$ higher insulin levels two hours after the OGTT administration when compared to the reduced function alleles. Overall, the information derived from this study demonstrates that polymorphisms of OCT1 genes may modulate an individual's clinical metformin response in humans. However, further studies are needed to replicate this effect in larger populations and in various ethnic groups to determine if the effects can be generalized to the overall population of the reduced function allele carriers. It may also be beneficial if studies to correlate $S L C 22 A 1$ polymorphisms with metformin toxicity.

On the other hand, a study examining whether OCT1 or OCT2 polymorphisms had any impact on the efficacy of metformin had different outcomes than other work in the area. ${ }^{20}$ In the study, Japanese subjects $(n=33)$ were categorized based on response to greater than three months of metformin therapy (24 responders and 9 nonresponders) and no remarkable differences in allelic frequency and hepatic OCT1 mRNA levels among the two groups were revealed, with the exception of the 408 Met allele. ${ }^{20}$ The $408 \mathrm{Met}$ allele showed a tendency to be higher in the nonresponders and also corresponded to a lower level of hepatic OCT1 mRNA levels, but this difference was deemed not significant. ${ }^{20}$ When OCT2 was analyzed, investigators found that OCT2 gene variation occurs less often than with other known drug transporters (ie, MDR1, MRP1, MRP2, OATP-C), which is an observation that is consistent with other research results. ${ }^{20}$ There were no significant differences in the prevalence of OCT2 gene variation among the two groups either. ${ }^{20}$
Possible reasons for the disagreement between Shikata and colleagues and Shu and colleagues may be contributed ethnic differences, small sample size $(n=33)$ and other concurrent medications (antihyperglycemics as well as others not listed) may have altered the participants response to metformin. The limitations of this study make it hard to arrive at a definitive conclusion, which necessitates further work in this area to confirm whether OCT1 is a mediator of metformin response.

\section{Role of OCT2 polmorphisms in determining metformin response}

Polymorphisms in the OCT2 gene have also been identified and shown to cause inter-patient variability in the pharmacokinetic and pharmacodynamic profile of metformin. ${ }^{18,23}$ Leabman and colleagues showed that four gene variants (M165I, A270S, R400C, K432Q) showed altered uptake of 1-methyl-4-phenylpyridinium (MPP+), which is a high-affinity substrate for OCT2 [18, 23]. Three (M165I, A270S, R400C) of the four showed revealed a reduced MPP + uptake capacity and the other (K432Q) showed an increased uptake activity. ${ }^{18}$ Two studies have been done to show the relationship between metformin clearance in individuals carrying three variants $(\mathrm{c} .596 \mathrm{C}>\mathrm{T}$, c.602C $>\mathrm{T}$, c. $808 \mathrm{G}>\mathrm{T}$ ) of the $S L C 22 A 2$ gene. One study was done in Korean subjects $(n=26)$ and the other was done in Chinese subjects $(n=15)$, but both studies showed that metformin clearance was reduced and plasma metformin concentrations were increased in carriers of these variants. ${ }^{23,29}$ However, the study using Chinese subjects also demonstrated that drugdrug interactions between metformin and cimetidine also depends on these polymorphisms..$^{29}$ Results with concomitant cimetidine use showed that renal secretion and clearance of metformin were reduced in for all variants, but particularly reduced for the homozygous TT variant carriers (18.7\% of the normal metformin clearance). ${ }^{29}$ Together, the results from these studies exemplify that the clearance and plasma concentrations of metformin is influenced by OCT2 polymorphisms and dose modifications based on the particular variants may be beneficial to maximize the efficacy and minimize the toxicity of metformin. ${ }^{23,29}$ However, there are no studies that relate $S L C 22 A 2$ polymorphisms with metformin toxicity (ie, lactic acidosis) nor is there enough evidence to develop a dose-adjustment scheme to manage patients with these OCT2 variants. Although the results are consistent between the two studies, further investigation with larger and more diverse sample populations need to be done to confirm these results and determine the need for developing a dose-adjustment 
algorithm for managing these patients. Additional studies to determine the specific structural-activity relationships associated with these polymorphisms may provide useful information on how to manage these polymorphisms to optimize metformin therapy.

\section{Role of MATEI polymorphisms in metformin response}

Becker and colleagues. looked at one polymorphism of the SLC47A1 gene (rs2289669G > A) and the associated reduction in $\mathrm{HbA}_{1 \mathrm{C}}$ in subjects $(\mathrm{n}=116)$ treated with metformin between 1997 and 2008..$^{22}$ The results showed that with each A-allele came a $0.3 \%$ larger reduction in $\mathrm{HbA}_{1 \mathrm{C}}(0.6 \%$ larger reduction than expected for homozygous rs2289669AA) after a 30-day treatment with metformin compared to the reference allele. ${ }^{22}$ In contrast, the homozygous $\mathrm{G}$ allele showed no reduction in the same context. ${ }^{22} \mathrm{HbA}_{1 \mathrm{C}}$ changes were based upon a value within 90 days (with an average time prior metformin initiation of 12 days and a standard deviation of \pm 16 days) of starting metformin therapy and 30 days after continuous metformin use. ${ }^{22}$ This demonstrates that SNPs in the SLC47A1 may affect the glucose-lowering affects of metformin. ${ }^{22}$ However, this was the first study to assess this association and further studies are needed to replicate this information in larger and diverse populations to determine if there are any associated clinical implications.

\section{Sulfonylureas}

\section{Role of $K_{\text {ATP }}$ channels in stimulating} insulin secretion in pancreatic $\beta$-cells

Pancreatic $\beta$-cells are equipped with ATP-sensitive potassium channels ( $\mathrm{K}_{\mathrm{ATP}}$ channels) that close in response to elevated cytosolic ATP/ADP concentrations, produced from glucose metabolism in the setting of elevated blood glucose. These $\mathrm{K}_{\text {ATP }}$ channels are composed of a Kir6.2 pore and SUR subunits, which regulate the open and closure status of the Kir6.2 pore based on ATP levels and is the target for the SU drug class. ${ }^{7,30}$ Closure leads to membrane depolarization and opening of intracellular voltage-gated $\mathrm{Ca}^{2+}$ channels that results in an increase in intracellular $\left[\mathrm{Ca}^{2+}\right]$, which stimulates insulin vesicle exocytosis and insulin release to reduce blood glucose. . $^{7,10,30,31}$

\section{Sulfonylurea mechanism of action}

Sulfonylurea exert their pharmacological actions through specifically binding the regulatory SU receptor (SUR1) subunit of the $\mathrm{K}_{\text {ATP }}$ channel to induce transformation to the closed state; thereby promoting insulin secretion and reduction in blood glucose. ${ }^{31}$ In addition, research has also proposed that SUs may also bind to the SUR2A subunit on peripheral $\mathrm{K}_{\text {ATP }}$ channels to increase glucose uptake in skeletal muscle and adipose tissues, but this action remains to be defined. ${ }^{31}$

\section{Sulfonylureas' place in therapy and possible reasons for interindividual variability in response}

The SUs are an alternative to metformin as a first-line option for the management of T2D. ${ }^{7}$ However, between $10 \%$ and $20 \%$ of patients experience a condition referred to as primary failure of SU treatment, which is characterized by a reduction in insulin secretion within the first three months of initiation. ${ }^{16}$ Explanations for this variability include polymorphisms in the genes that encode for the Kir6.2 and SUR1 subunits (KCNJ11 and $A B C C 8$, respectively) of the $\mathrm{K}_{\mathrm{ATP}}$ channel and in metabolizing enzyme, CYP2C9. ${ }^{30}$

Several studies demonstrate genetic variations among the genes that encode for the Kir6.2 pore (KCNJ11 gene) and the SUR1 subunits ( $A B C C 8$ gene) of pancreatic $\mathrm{K}_{\mathrm{ATP}}$ channels can alter response to $\mathrm{SU}$ therapy, but they were mainly observed in the neonatal diabetic population because that is where these polymorphisms are predominately found..$^{30,31}$ Neonatal diabetes is a monogenic form of diabetes typically diagnosed during the first three months of life and subdivided into a form that that usually reconciles after 18 months, transient neonatal diabetes (TND), and a lifelong form, persistent neonatal diabetes (PND). ${ }^{31}$ These forms of diabetes can be attributed to genetic variations in the $\mathrm{K}_{\text {ATP }}$ channel, which results in a decrease in ATP-sensitivity and/or a pore fixed in the open conformation. ${ }^{31}$ In general, $K C N J 11$ and $A B C C 8$ gene polymorphisms alter the $\mathrm{K}_{\text {ATP }}$ channels sensitivity to ATP or favor an open state leading to a reduction in insulin secretion and subsequent hyperglycemia in these individuals (Table 2). ${ }^{31-33}$

\section{$\mathrm{KCNJII}$ and $\mathrm{ABCC} 8$ polymorphisms in neonatal diabetes and the associated effects on sulfonylurea response}

One study categorizes the variations to those that directly affect ATP sensitivity and those that indirectly affect ATP sensitivity and examine the associated response to SU therapy. ${ }^{31}$ Genetic variations that directly affect ATP sensitivity (I182V; $A B C C 8$ ) do so by decreasing $\mathrm{K}_{\text {ATP }}$ channel affinity for ATP, which causes the $\mathrm{K}_{\mathrm{ATP}}$ channel to remain open that translates to a reduction in insulin secretion. ${ }^{31}$ 
Indirect genetic variations (Q52R, V59M, I296L; KCNJ11) increases the stability of the $\mathrm{K}_{\text {ATP }}$ channel in the open conformation, which has been previously shown to induce functional detachment of the SUR1 subunit from the poreforming Kir6.2 subunits. ${ }^{31}$ SUs block the conductance of $\mathrm{K}_{\text {ATP }}$ channels by interacting with the SUR1, referred to as the high-affinity site, as well as Kir6.2 subunits referred to as the low-affinity site. ${ }^{31}$ The functional detachment of the SUR1 from the Kir6.2 subunit leads to a decreased sensitivity to SUs (in this study, glibenclamide and tolbutamide) by reducing the high-affinity block. ${ }^{31}$ The results show that glibenclamide blocked the conductance $\mathrm{K}_{\mathrm{ATP}}$ channel almost completely in the wild-type, but the variant cells were relatively insensitive to glibenclamide and I296L carriers essentially unresponsive to glibenclamide treatment. With regard to tolbutamide treatment, mild reductions in current were seen in Q52R, V59M, and I183V, but I296L was completely insensitive to the effects of tolbutamide. These results indicate that individuals carrying the I296L variant are less sensitive to SU therapy and may require higher doses of SU therapy to achieve the same goals compared to the other three variants or may even require insulin therapy. However, the other three variants (Q52R, V59M, I183V) may simply need a higher SU dose to achieved goals and the dose would be dependent on the variant expressed. Although, a definitive dosing guide is not available presently, the rank of sensitivities to either SU (I182V > V59M > Q52R > I296L) may provide a general dosing algorithm to help adapt the dose to the particular variant.

There are several other KCNJ11 variants (which include F333I, F35V, R201H, R201C, Q52R, I296L, L164P, G53S, $\mathrm{G} 53 \mathrm{R}$ ) and $A B C C 8$ variants (which include I182V, H1023Y, I1424Y, F132L) that increase susceptibility to neonatal diabetes, but in the majority of the variant genotypes seem to have a preserved response to SU therapy with the exception of Q52R, I296L, and L164P variants. ${ }^{7,31,33-38} \mathrm{KCNJ} 11$ variant carriers may only need higher doses of SU therapy to achieve therapeutic goals. Since, SUs are not effective for all variant carriers genetic screening for these variants would be a valuable tool for selecting SU therapy candidates. ${ }^{7}$ Alternatively, further investigations are needed to develop a dosing algorithm for those with $K C N J 11$ and $A B C C 8$ polymorphisms that respond to SU therapy, but require dose modifications.

Patients with permanent neonatal DM and mutations affecting Kir6.2 response to SU. ${ }^{33,39}$ suggest that many of these patients may successfully be switched to oral drugs (such as SU) instead of insulin. Pearson and colleagues reported a $90 \%$ success rate (44 of 49 patients) in switching from subcutaneous insulin injections to oral glyburide. ${ }^{39}$ Babenko and colleagues had five of nine patients successfully switch from insulin injection to oral SU therapy with permanent neonatal DM, in these cases caused by mutations in the SUR1 subunit. ${ }^{32}$ This instance of patients with permanent neonatal DM with mutant Kir6.2 or SUR1 to SU's illustrates just how powerful genetics may be in identifying patients who may or may not benefit from a certain treatment. It may be of value to test all those with neonatal diabetes for mutations in Kir6.2 and SUR1.

\section{Role of KCNJII and ABCC8 polymorphisms in sulfonylurea response in type 2 diabetics}

A study by Feng and colleagues with a total of 1,268 Chinese $\mathrm{T} 2 \mathrm{D}$ patients was conducted. These patients were all recently diagnosed diabetics (within the past five years) and had had no recent hypoglycemic treatment. They were treated with gliclazide for eight weeks, and fasting and oral glucose tolerance test two-hour plasma glucose, fasting insulin and A1C were measured at the start and after eight weeks of treatment. After eight weeks of treatment it was found that patients with Ser1369Ala of the ABCC8 gene and rs5210 of the KCNJ11 gene had a significant decrease in FPG $(p=0.002)$. This was also confirmed in cohort 2 of the study and in the pooled analysis subjects with the Ala/Ala genotype had a $7.7 \%$ greater decrease in FPG $(p<0.001)$, a 3.5 greater decrease in $\mathrm{A} 1 \mathrm{C}(\mathrm{p}=0.06)$ and a $11.9 \%$ greater decrease in two-hour plasma glucose $(p=0.003)$ than those with the Ser/Ser genotype after eight weeks of gliclazide treatment. ${ }^{40}$

\section{Role of CYP2C9 polymorphisms in sulfonylurea response}

While genetic alterations in the transporting and target proteins can produce variability in response, drug metabolizing enzyme activity may also contribute the response variability. With respect to four United States available (Tolbutamide [Orinase ${ }^{\circledR}$ ], Glipizide [Glucotrol ${ }^{\circledR}$ ], Glibenclamide [Glyburide ${ }^{\circledR}$ ] and Glimepiride (Amaryl $\left.{ }^{\circledR}\right]$ ) SUs, the major metabolizing enzyme is cytochrome P450 2C9 (CYP2C9). ${ }^{10}$

Kirchheiner and colleagues investigated three allelic forms (CYP2C $9 * 3 / * 2 / * 1)$ of CYP2C9 and compared there hepatic metabolism to the wild-type (CYP2C $9 * 1 / * 1) .{ }^{10}$ The CYP2C $9 * 3 / * 3$ revealed lower clearances of tolbutamide, glibemclamide, and glipizide (16\%, 50\%, and $20 \%$, respectively) compared to that of the wild-type. ${ }^{10}$ Homozygous CYP2C $9 * 2 / * 2$ showed a mildly reduced clearance of tolbutamide and 
glibemclamide ( $75 \%$ and $90 \%$, respectively) compared to the wild-type, but this difference was not deemed statistically significant. ${ }^{10}$ Heterozygous condition CYP2C $9 * 1 / * 2$ showed a similar clearance with respect to the wild-type for tolbutamide and allele $* 2$ carriers showed a mildly reduced clearance for glibenclamide compared to the wild-type (homozygous $* 2 / * 2$ carriers showed $90 \%$ clearance compared to wild-type). Both heterozygous CYP2C $9 * 1 / * 3$ and CYP2C $9 * 2 / 3$ showed a $50 \%$ reduction in the clearance of tolbutamide, but and the reduction was less pronounced for glibenclamide. Overall, the wild-type, CYP2C $9 * 2 / * 2$, and $* 1 / 22$ genotypes showed roughly normal (CYP2C $9 * 2 / * 2)$ to extensive (CYP2C $9 * 1 / 2)$ clearance rates, CYP2C $9 * 1 / * 3$ and $* 2 / * 3$ genotypes showed intermediate clearance rate and the $\mathrm{CYP} 2 \mathrm{C} 9 * 3 / * 3$ genotype clearance rate was significantly reduced in participants. This data suggests that pre-screening individuals for these polymorphisms may help guide dosing, which may limit toxicity in these individuals and maximize efficacy; although, correlations between enzyme activity and toxicity was not demonstrated making it hard to rationalize dose adjustments. Therefore, more studies replicating this data in larger and ethnically diverse populations are needed.

In a second study, Caucasian volunteers $(n=475)$ expressing CYP2C9 variable function alleles and receiving SU therapy (tolbutamide $62.3 \%$, glibenclamide $16.2 \%$, glimepiride $16 \%$, glicazide $5.5 \%$ ) were analyzed based on changes in dose from their first prescription to the tenth prescription and changes in blood glucose in a period 90 days before and 180 days after beginning SU therapy $(n=79){ }^{41}$ In contrast to the previous study, clinically relevant variation in SU pharmacokinetics and response in participants with different CYP2C9 polymorphisms were only found in the tolbutamide group and not in any other treatment groups ${ }^{41}$ The tolbutamide group carrying the $* 3$ allele showed only a minute change in dose between the first and the tenth prescriptions compared to that of the wild-type
(CYP2C9*1/*1; $12 \mathrm{mg}$ versus $279 \mathrm{mg}$, respectively). ${ }^{41}$ Additionally, 15 of the 20 individuals expressing the *3 allele receiving tolbutamide showed no change in dose from the first prescription to the tenth prescription. ${ }^{41}$ No differences between tolbutamide group expressing the $* 1 / * 2$ and $* 2 / * 2$ genotypes were found compared to the wild-type group, indicating that that metabolism was not necessary in these individuals, which is consistent with Kirchheiner and colleagues. In a supplementary investigation, the change in dose from the sixth to twentieth prescription was compared to the first prescription using repeated measurements. ${ }^{41}$ These results showed that $* 3$ allele carriers receiving tolbutamide showed a daily prescribed dose of $316 \mathrm{mg}$ lower compared to the wild-type tolbutamide group $(\mathrm{p}=0.0008) .{ }^{41}$ Lastly, in the secondary objective analysis (that measured changes in FPG between a period 90 days prior and 180 after staring SU therapy) no reductions in the tolbutamide groups, regardless of allele, compared to the wild-type reached statistical significance. The findings of this data clearly show that there is a relationship between the CYP2C $9 * 3$ allele carriers and their corresponding prescribed dose of tolbutamide, but this relationship may not be extended to the glucose lower potential of SU therapy ${ }^{41}$ However, the relationship between CYP2C9 polymorphism and SU toxicity may have clinical relevance and should be considered in future studies. ${ }^{4}$

\section{Role of TCF7L2 variation in sulfonylurea response}

Transcription factor 7-like 2 (TCF7L2) variants are associated with T2D risk, it is thought due to decreased $\beta$-cell function. A study by Pearson and colleagues in which 4,469 T2D patients were determined to have the TCF7L2 genotype, of these 901 were incidentally being treated with SU and 945 with metformin. A1C pretreatment was available for a subset of these patients (579 of the patients treated with SU and 755 of those on metformin). A logistic regression was used

Table 2 Possible role and effects of variations on sulfonylurea treatment goals

\begin{tabular}{|c|c|c|c|}
\hline & KCNJI I & ABCC8 & CYP2C9 \\
\hline Codes & Kir6.2 & SURI & Enzyme in cytochrome $\mathrm{P} 450$ \\
\hline Site & $\mathrm{K}_{\text {ATP }}$ channels of pancreas & & Liver \\
\hline Action & $\begin{array}{l}\text { SU blocks conductance of } K_{\text {ATP }} \text { channels by interacting } \\
\text { with SURI and Kir6.2 subunits }\end{array}$ & & Metabolize SU \\
\hline Variants & $\begin{array}{l}\text { Decreased ATP sensitivity and/or pore fixed in the } \\
\text { open conformation leading to decreased insulin } \\
\text { secretion and subsequent hyperglycemia. Different } \\
\text { variations result in different sensitivities to SU }\end{array}$ & $\begin{array}{l}\text { Ser I369Ala variant can } \\
\text { influence SU efficacy }\end{array}$ & $\begin{array}{l}\text { Reduced clearance which } \\
\text { may correlate to SU toxicity }\end{array}$ \\
\hline
\end{tabular}

Abbreviations: ATP, adenosine triphosphate; SU, sulfonylurea. 
and treatment failure was defined as $\mathrm{A} 1 \mathrm{C}$ of $>7 \%$ within 3-12 months of treatment initiation. The study found that carriers of the risk allele were less likely to respond to SU's (odds ratio [OR] for failure $1.95 \% ; \mathrm{p}=0.005$, comparing $\mathrm{rs} 12255372$ $\mathrm{T} / \mathrm{T}$ vs G/G). When the A1C baseline was included the association was strengthened (OR 2.16; $p=0.009)$. In conclusion the researchers found that TCF7L2 variants did in fact influence patient response to SUs, but not metformin. ${ }^{42}$

\section{Thiazolidinedione}

\section{Thiazolidinedione place in therapy}

The TZDs, otherwise referred to as glitazones, are included in the battery of type 2 antidiabetic agents that are prescribed as an adjunct to diet and exercise (monotherapy) or in combination with SUs, metformin, or insulin. ${ }^{43}$ The TZDs currently available to the United States market are pioglitazone $\left(\operatorname{Actos}^{\circledR}\right.$ ) and rosiglitazone (Avandia $\left.{ }^{\circledR}\right){ }^{43}$ TZDs have shown to increase insulin sensitivity of myocytes, adipocytes, and hepatocytes as well as reducing FPG and $\mathrm{HbA}_{1 \mathrm{C}}$ levels in several, but not all individuals. ${ }^{43}$ An estimated $20 \%$ and $30 \%$ of individuals fail to reach goal insulin sensitivity and glycemic measures following TZD therapy. ${ }^{43}$ Studies have shown that the interindividual variability in TZD response may be attributed, at least partially, to genetic inheritance. ${ }^{43}$ Genetic polymorphisms in TZD target proteins, effector proteins, and metabolizing enzymes have been shown to contribute to variability in response to TZDs in a number of studies (Table 3$).^{43}$

\section{Thiazolidinedione mechanism of action}

TZDs pharmacologically act by agonizing the nuclear perixisome proliferator-activated receptor gamma (PPAR- $\gamma$ )

Table 3 Possible role and effects of variations on TZD therapy

\begin{tabular}{lll}
\hline $\begin{array}{l}\text { TZD } \\
\text { therapy }\end{array}$ & ACDC & S447S \\
\hline Action & $\begin{array}{l}\text { Adiponectin } \\
\text { Protective, plays role in glucose } \\
\text { uptake and fatty acid oxidation. } \\
\text { Decrease in adiponectin } \\
\text { associated with insulin } \\
\text { resistance and atherosclerosis }\end{array}$ & $\begin{array}{l}\text { LPL } \\
\text { and mediate clearance of } \\
\text { atherogenic lipoproteins }\end{array}$ \\
Variants & $\begin{array}{l}\text { +45GG allele smaller decreases } \\
\text { in FPG level, AIC and increase } \\
\text { in adiponectin than those with } \\
+45 T \text { allele }\end{array}$ & $\begin{array}{l}\text { S447X shows reduced } \\
\text { response }\end{array}$ \\
& $\begin{array}{l}\text { Similarly the }+276 G G \\
\text { genotype when compared to } \\
\text { the }+276 T \text { allele }\end{array}$ & \\
\hline
\end{tabular}

to form heterodimers with the retinoid $\mathrm{X}$ receptors (RXR), which binds the PPAR-response elements and regulates target gene transcription. ${ }^{43}$ The heterodimer (PPAR- $\gamma+$ RXR) complex plays a role in regulating the transcription of genes involved in fatty acid uptake and storage, glucose uptake, adipocyte differentiation, and cytokine production and was recently shown to regulate the expression OCT1 via the fatty acid binding protein 2 (FABP2) gene. ${ }^{43,44}$

\section{Role of PPAR- $\gamma$ in determining type 2 diabetes susceptibility}

Discovery of a polymorphism in the PPAR- $\gamma 2$, Pro12Ala, found mainly in the adipocytes has been studied extensively in attempt to provide some plausible insight into TZD interindividual response variability. ${ }^{43,45}$ It is estimated that Pro12Ala has a frequency of roughly $12 \%$ to $15 \%$ in the general population, making it the most common PPAR- $\gamma$ variant. ${ }^{46}$ Two functional studies show that the TZD-activated PPAR- $\gamma 2$ Ala1 2 allele exhibited a reduced binding affinity for the PPAR promoter region..$^{47,48}$ A meta-analysis showed that Ala12 allele displayed a protective effect with regard to developing T2D, reducing the risk in carriers by $19 \%{ }^{45}$ However, the risk reductions were more pronounced in Asians (35\%) than in North Americans (18\%) or Europeans $(15 \%)$, which progressively diminished from northern $(10 \%)$ to southern Europe ( $0 \%)$. Furthermore, the protective effect of the Ala12 allele appeared to be inversely related to body mass index..$^{45}$

\section{Role of PPAR- $\gamma$ polymorphisms in thiazolidinedione response}

The question of how PPAR- $\gamma$ polymorphisms affects response to TZD therapy was addressed by Bluher and colleagues and Kang and colleagues, but yielded inconsistent results. ${ }^{46,49}$ Bluher and colleagues conducted a study in German T2D subjects $(\mathrm{n}=131)$ using pioglitazone and determined that there was no significant difference in response to piogltiazone between the heterozygous genotype (Pro12Ala) and the homozygous genotype (Pro12Pro). ${ }^{46}$ With regard to the homozygous carriers, the study was underpowered to detect a difference between the response rates compared to Pro12Ala and Pro12Pro genotypes. ${ }^{46}$ Additionally, individuals with heterozygous and homozygous Pro12 genotypes showed a significant $(\mathrm{p}<0.05)$ reduction in triglycerides and carriers of the Ala12Ala genotype showed a significant $(p<0.05)$ elevation in high-density lipoprotein levels after six months of pioglitazone therapy. ${ }^{46}$ On the other hand, Kang and colleagues conducted a similar study 
in Korean T2Ds receiving rosiglitazone for 12 weeks and found the response rate of the Pro12Al genotype was significantly $(\mathrm{p}=0.002)$ higher in comparison to the homozygous Pro12 genotype ( $86.7 \%$ and $43.7 \%$, respectively). ${ }^{49}$ As in the previous study, the homozygous Ala12 was not observed. ${ }^{49}$ Based on the results of each study, it would be hard to arrive at a convincing conclusion to explain if response rates depend on Pro12Al or not. Potential factors leading to inconsistent results may be attributed to differences in ethnicity, environmental factors, selected TZDs, and differences in baseline characteristics. ${ }^{49}$ Additionally, insulin sensitivity was restored to a greater degree in the heterozygous (Pro12Ala) versus the homozygous (Pro12Pro) participants, which was inconsistent with other studies using troglitazone for 12 weeks and one year. ${ }^{49-51}$ Possible factors leading to inconsistencies between these three trials include differences in the ethnicity of study populations, treatment drug and duration and method for measuring insulin sensitivity.

\section{Role of adiponectin in thiazolidinedione response}

Alternatively, effector protein, adiponectin, was shown to be increased in individuals receiving TZD treatment and found to play a role in the insulin-sensitizing effect of TZDs. ${ }^{52}$ Encoded by the $A C D C$ gene, adiponectin is secreted into circulation from the adipose tissue and has been seen to be associated with insulin resistance and atherosclerosis in low systemic concentrations and is protective at high concentrations. ${ }^{52}$ This may be contributed to the fact that adiponectin modulates metabolic processes, including glucose uptake and fatty acid oxidation. ${ }^{43}$ Studies have revealed several SNPs in the encoding gene, $A C D C{ }^{52}$ Of those that were discovered, three (SNP45, SNP276, SNP11377) are associated with T2D, circulating adiponectin levels, and insulin resistance in Japanese individuals and two (SNP11391, SNP11377) in French individuals. ${ }^{52}$ One study examined the effects of two SNPs (SNP45 [TT/GG/TG], SNP276 [TT/GG/TG]) on the response rate in Korean individuals receiving rosiglitazone $4 \mathrm{mg}$ for 12 weeks. ${ }^{52}$ The result indicated rosiglitazone significantly reduced FPG levels, $\mathrm{HbA}_{1 \mathrm{C}}$ levels and significantly raised adiponectin levels after 12 weeks, without considering genotype. ${ }^{52}$ Taking into account the individual SNPs and genotypes of the individuals, investigators found that carriers of the $+45 \mathrm{GG}$ genotype had significantly smaller decreases in FPG level, $\mathrm{HbA}_{1 \mathrm{C}}$ and increases in adiponectin levels compared to those who carried the $+45 \mathrm{~T}$ allele. ${ }^{52}$ Similarly, the +276 GG genotype showed the same profile when compared to the $+276 \mathrm{~T}$ allele participants. ${ }^{52}$ Furthermore, the 45/276GG haplotype followed suit in showing smaller reductions in FPG and $\mathrm{HbA}_{1 \mathrm{C}}$ and smaller increases in adiponectin levels relative to the other haplotypes following rosiglitazone therapy in the 166 subjects tested. ${ }^{52}$ In conclusion, the $45 / 276 \mathrm{GG}$ haplotype can reduce an individual's response to rosiglitazone therapy and may be a clinically relevant consideration for rosiglitazone initiation, but the question of whether these results can be extrapolated to pioglitazone remains to be elucidated along with the functional mechanism by which this occurs.

\section{Role of lipoprotein lipase in thiazolidinedione response}

Lipoprotein lipase (LPL) is another effector protein of TZD response and the expression has been shown to increase after pioglitazone treatment. ${ }^{43} \mathrm{LPL}$ is an enzyme that functions to hydrolyze triglyceride and mediate the clearance of atherogenic lipoproteins. $\mathrm{A} \mathrm{C} \rightarrow \mathrm{G}$ polymorphism at the position 1595 in the $L P L$ gene results in a premature stop codon at number 447 , referred to as $\mathrm{S} 447 \mathrm{X} .{ }^{43}$ In one study, the association between the $\mathrm{S} 447 \mathrm{X}$ polymorphism and pioglitazone response was analyzed in Chinese individuals with T2D. ${ }^{43}$ The results of this study indicated that the homozygous genotype, S447S, was associated with a higher percentage of patients achieving the response criteria compared to the other genotypes. ${ }^{43}$ In contrast, the $\mathrm{S} 447 \mathrm{X}$ show a 0.538 -fold reduction in response to pioglitazone treatment compared to the $\mathrm{S} 447 \mathrm{~S}$ genotype. ${ }^{43}$ This suggests that $L P L$ polymorphisms are a useful predictor in determining how well an individual will respond to pioglitazone therapy.

\section{Common polymorphisms associated with thiazolidinedione toxicity}

While efficacy is an important part of drug response, toxicity may negate the efficacy benefits in some cases making it imperative that it is considered along with the efficacy component. TZDs have displeasing adverse effects, which often outweigh the utility of their benefits and limit their use. Adverse events include weight gain, edema, heart failure, bone fractures in women, and idiosyncratic hepatotoxicity. ${ }^{43}$

\section{Perilipin and thiazolidinedione-induced weight gain}

Weight gain was addressed in a study evaluating the SNPs in the candidate gene, perilipin. ${ }^{43}$ Four common polymorphisms (6209G/A, 11482G/A, 13041A/G, 14995A/T) were 
examined in this study. ${ }^{43}$ Results showed that the $11482 \mathrm{G} / \mathrm{A}$ variant is significantly associated with weight gain following 12 weeks of rosiglitazone therapy. ${ }^{43}$ The most pronounced weight gain occurred in the homozygous $11482 \mathrm{G} / \mathrm{G}$ carriers relative to $\mathrm{G} / \mathrm{A}$ and $\mathrm{A} / \mathrm{A}(1.33,0.85$, and $0.03 \mathrm{~kg}$, respectively). ${ }^{43}$ This suggests that $\mathrm{A} / \mathrm{A}$ genotype carriers are less likely to exhibit weight gain. ${ }^{43}$

\section{Role of Prol 2Ala genotype in thiazolidinedione-induced edema}

In terms of edema, there is one study that association between the Pro12Ala polymorphism and edema risk in T2Ds. ${ }^{43}$ Ragalitazar is a dual PPAR- $\gamma$ and PPAR- $\alpha$ activator that improves FPG and $\mathrm{HbA}_{1 \mathrm{C}}$ comparable to pioglitazone, but displays more positive effects on the lipid profile on a study performed on 177 subjects. ${ }^{53}$ Data from this study showed a 4.4-fold decrease in the risk of edema in participants carrying the Pro12Pro genotype compared to those that had the Ala12 allele, suggesting that the Ala12 may be less prone to TZD-induced edema. However, whether these results may be extrapolated to the TZDs remains to be determined, even when it is known that ragaglitazar acts via a similar mechanism. ${ }^{43}$

\section{Role of CYP2C9 polymorphisms in thiazolidinedione-induced hepatotoxicity} A Japanese retrospective study looked at the role of CYP2C19 or CYP2D6 enzyme polymorphisms in troglitazone-induced hepatotoxicity in a study that aimed to define the cause of TZD-induced hepatotoxicity. ${ }^{43}$ Retrospective analyses revealed that the group with troglitazoneinduced hepatotoxicity was comprised of a larger percentage of CYP2C19-poor metabolizers compared to the group who did not experience hepatotoxicity. ${ }^{43}$ However, rosiglitazone and pioglitazone have a much lower likelihood of causing TZD-induced hepatotoxicity making it hard to generalize the results to them. ${ }^{43}$ Lastly, the role of CYP2C19 in troglitazone metabolism was also undetermined. ${ }^{43}$

A chief contributor in the development of any to concentration-dependent toxicity is the integrity of an individual's metabolizing enzymes. TZDs are mainly metabolized by CYP2C8, but CYP2C9 and CYP3A4 also contribute to lesser extents. ${ }^{43}$ Rosiglitazone and pioglitazone are primarily metabolized by CYP2C8, but CYP2C9 and CYP3A4 contribute to a lesser extent in the metabolism of rosiglitazone and pioglitazone, respectively. ${ }^{10,43}$ The studies that looked at the association between CYP2C8 polymorphisms and TZD toxic- ity are inconsistent and generally did not produce statistically significant results. ${ }^{10}$ Therefore, it can only be speculated that polymorphisms in TZD-metabolizing enzymes are associated with toxicity.

\section{Meglitindes \\ Use of meglitinides and their mechanism of action}

The antidiabetic drug class of meglitinides is comprised of nateglinide $\left(\right.$ Starlix $\left.^{\circledR}\right)$ and rapeglinide $\left(\operatorname{Prandin}^{\circledR}\right) .{ }^{54}$ Similar to the SUs, the meglitinides act on the $\mathrm{K}_{\mathrm{ATP}}$ channel, but at a distinct binding site, to induce insulin secretion as well as on calcium voltage-gated channels. ${ }^{10}$ They stimulate earlyphase insulin production to reduce postprandial glucose and their effects are short-lived and leave late-phase and fasting insulin untouched. ${ }^{10}$ Possible reasons for interindividual variability in response to this class of antidiabetic medications can be contributed to polymorphisms in organic anion transporting polypeptide 1B1 (OATP1B1) gene $(S L C O 1 B 1)$ or the metabolizing enzyme, CYP2C9 (Table 4). ${ }^{55,56}$

\section{Role of SLCOIBI polymorphisms in meglitinide response}

Zhang and colleagues studied three SNPs (521TT, 521TC, $521 \mathrm{CC}$ ) of the SLCO1B1 gene and their associated effect on the pharmacokinetics of nateglinide. ${ }^{55}$ The results showed carriers of heterozygous 521TC and homozygous 521CC had significantly higher plasma concentrations (83\% and $76 \%$, respectively) of nateglinide compared to the homozygous TT carriers. ${ }^{55}$ The half-life of nateglinide was $78 \%$ longer $(\mathrm{p}=0.036)$ in individuals carrying the 521CC SNP compared to the 521TT carriers. ${ }^{55}$ Lastly, the AUC in the 521TC and $521 \mathrm{CC}$ carriers were significantly higher $(82 \%$ and $108 \%$, respectively) than that of the $521 \mathrm{TT}$ carriers. ${ }^{55}$ These results suggest that $S L C O 1 B 1$ polymorphisms have a significant impact on the plasma concentrations of nateglinide, which

Table 4 Possible role and effects of variations on meglitinide therapy

\begin{tabular}{lll}
\hline & SLCO IB I & CYP2C9 \\
\hline Action & $\begin{array}{l}\text { Possible role in hepatic uptake } \\
\text { (transporter) }\end{array}$ & Metabolization \\
Variants & 52ITC, 52 ICC significantly & Reduced metabolizing \\
& higher plasma concentrations \\
compared to homozygous & activity in some \\
& variations \\
& 52ICC SNP compared to 52 ITT & \\
\hline
\end{tabular}


may be contributed to a reduced hepatic uptake. ${ }^{55}$ However, the hepatic uptake, efficacy, and toxicity associated with the elevated plasma levels of nateglinide were not assessed, which makes it hard to apply this data clinically, but may act as a starting point for genetics-based nateglinide-prescribing practice.

Another study that assessed the effect of SLCO1B1 polymorphisms and the associated effects on nateglinide and rapeglinide pharmacokinetics produced conflicting results with respect to nateglinide. ${ }^{56}$ Results showed that the $521 \mathrm{~T}>\mathrm{C}$ polymorphisms of SLCO1B1 does not alter the pharmacokinetics and efficacy (ability to reduce blood glucose) of nateglinide or its metabolite (M7), regardless of the individual's genotype.$^{56}$ In contrast, the pharmacokinetics of repaglinide and its M2 and M4 metabolites were significantly affected by the $521 \mathrm{~T}>\mathrm{C}$ polymorphism. ${ }^{56}$ Individuals expressing the 521CC genotype tended to have higher plasma concentrations of repaglinide and its metabolites, which also correlated with a greater ability to reduce blood glucose. ${ }^{56}$ This indicates that the 521CC genotype may encode for a reduced function OATP1B1 transporter. Although the incidence of adverse events was not assessed in either study, this may be an important avenue for future studies to determine the clinical relevance this polymorphism.

\section{Role of CYP2C9 in meglitinde metabolism and associated effect on response}

Nateglinide is metabolized by CYP2C9 and repaglinide is metabolized by CYP2C8. Polymorphism of CYP2C9 were addressed in the same study that addressed the SU and was conducted using the same allelic forms (CYP2C $9 * 1 / * 2 / * 3) .{ }^{10}$ Heterozygous forms, CYP2C $9 * 1 / * 3$ and CYP2C $9 * 2 / * 3$, and homozygous forms, CYP2C9*2/*2, exhibited intermediately reduced metabolizing activity compared to the wild-type (CYP2C9*1/*1). ${ }^{10}$ However, similar to the SUs, CYP2C $9 * 3 / * 3$ showed a significantly reduced $(50 \%)$ metabolizing activity compared to the wild-type. ${ }^{10}$ A population pharmacokinetic/pharmacodynamics model was used to extrapolate associations between nateglinide and plasma insulin and glucose concentrations. Results showed that the risk for hypoglycemia increases for doses of $180 \mathrm{mg}$ and higher and individuals with the CYP2C9*3/*3 genotype demonstrated an enhanced risk. ${ }^{10}$ Although the recommended upper limit is $120 \mathrm{mg}$, caution should be used in the homozygous CYP2C9*3 carriers. ${ }^{10}$ This information is inadequate to make dose-adjustment recommendations and carriers of these genotypes should be evaluated further to assess associated response and toxicity. On the other hand, a study investigating the CYP2C8 polymorphisms revealed highly contradictory results, which makes it hard to see a definitive effect with repaglinide. ${ }^{10}$ Other studies are warranted to examine the association between repaglinide and CYP2C8 polymorphisms.

\section{Discussion}

Pharmacogenomics has become a revolutionary approach for defining an individual's clinical response to drug therapy by permitting researchers to investigate most, if not all, patient genomes for insight. However, there are still many ethical, social, economic, legislative, and research protocol issues that need to be sorted out before the promise of "personalized medicine" become a customary approach clinical practice. Furthermore, a great deal of education for the public and health care professionals in the area is necessary before gaining overall acceptance. Nevertheless, advances are rapidly moving this approach closer to clinical practice to be implicated in the management of many complex diseases (ie, cardiovascular disease, cancer, and DM).

Pharmacogenomic investigations of T2D have yielded variations in genomic loci containing common variants with translational consequences of altering disease susceptibility risk, clinical course, and response to standard therapy. Discoveries of gene polymorphisms in drug transporter, targets, effector proteins, and metabolizing enzymes have only served to augment discoveries of possible causal genes. Therefore, it is important to keep pushing the pharmacogenomic approach to advance our knowledge in disease state prevention and management to attain optimal health outcomes.

\section{Executive summary}

Inquiry regarding disease prevention, therapy and prognosis adopted an approach that interrogates the entire genome (genomics) for answers versus single genes (genetics), which has facilitated the discovery of gene variations in the form of SNPs in several genes associated with complex disease states.

One of the many areas in complex disease state management that the genomic approach has offered great insight is in T2D pharmacotherapy. Specifically, pharmacogenomics has provided a greater understanding of biological mechanisms that cause or contribute to interindividual variability in response of oral antidiabetic therapy. OCT1, OCT2, and MATE1 SNPs have shown to alter metformin's pharmacokinetics and ability to inhibit glucose output from the liver and may help explain variability in response. 
SNPs in genes encoding the subunits of the $\mathrm{K}_{\text {ATP }}$ channel and hepatic metabolizing enzyme CYP2C9 have been shown to alter individual sensitivity to SU therapy, whereas polymorphisms in CYP2C9 has been shown to alter the pharmacokinetics.

Efficacy of thiazolidinedione therapy appears to be enhanced in individuals expressing genes resulting in higher levels of adiponectin and lipoprotein lipase. Additionally, SNPs in PPAR- $\gamma$ have the potential to either enhance or reduce patient response to TZD therapy. Altered response to meglitinide therapy is largely due to SNPs that result in a lower functioning OATP1B1 transporter, resulting in reduced hepatic uptake or polymorphisms in metabolizing enzymes.

Ethical, political, legislative, economic, and technological issues have delayed widespread acceptance of pharmacogenomics into clinical practice. However, these issues are being resolved at a fairly rapid pace, which puts pharmacogenomics at the forefront of future medicine in clinical practice.

\section{Disclosures}

The authors report no conflicts of interest in this work.

\section{References}

1. Khoury MJ, Valdez R, Albright A. Public health genomics approach to type 2 diabetes. Diabetes. 2008;57(11):2911-2914.

2. McCarthy MI, Zeggini E. Genome-wide association scans for type 2 diabetes: new insights into biology and therapy. Trends Pharmacol Sci. 2007;28(12):598-601.

3. Khoury MJ. Genetics and genomics in practice: the continuum from genetic disease to genetic information in health and disease. Genet Med. 2003;5(4):261-268.

4. Epstein RS, Frueh FW, Geren D, et al. Payer perspectives on pharmacogenomics testing and drug development. Pharmacogenomics. 2009;10(1):149-151.

5. Pearson TA, Manolio TA. How to interpret a genome-wide association study. JAMA. 2008;299(11):1335-1344.

6. Sadée W, Dai Z. Pharmacogenetics/genomics and personalized medicine. Hum Mol Genet. 2005;14 Spec No. 2:R207-R214.

7. Sattiraju S, Reyes S, Kane GC, Terzic A. K(ATP) channel pharmacogenomics: from bench to bedside. Clin Pharmacol Ther. 2008;83(2): 354-357.

8. Zhang W, Huang S, Dolan E. Integrating epigenomics into pharmacogenomic studies. Pharmacogenomics and Personalized Medicine. 2008;1:7-14.

9. Perry JR, Frayling TM. New gene variants alter type 2 diabetes risk predominantly through reduced beta-cell function. Curr Opin Clin Nutr Metab Care. 2008;11(4):371-377.

10. Kirchheiner J, Roots I, Goldammer M, Rosenkranz B, Brockmoller J. Effect of genetic polymorphisms in cytochrome p450 (CYP) 2C9 and CYP2C8 on the pharmacokinetics of oral antidiabetic drugs: clinical relevance. Clin Pharmacokinet. 2005;44(12):1209-1225.

11. John Wiley \& Sons, Ltd. Current literature in diabetes. Diabetes Metab Res Rev. 2009; Aug 27. DOI: 10.1002/dmrr.

12. Neeli H, Gadi R, Rader DJ. Managing diabetic dyslipidemia: beyond statin therapy. Curr Diab Rep. 2009;9(1):11-17.

13. American Diabetes Association. Economic costs of diabetes in the U.S. In 2007. Diabetes Care. 2008;31(3):596-615.
14. Misra A, Khurana L. Obesity and the metabolic syndrome in developing countries. J Clin Endocrinol Metab. 2008;93(11 Suppl 1):S9-S30.

15. Ripsin CM, Kang H, Urban RJ. Management of blood glucose in type 2 diabetes mellitus. Am Fam Physician. 2009;79(1):29-36.

16. Wolford JK, Vozarova de Courten B. Genetic basis of type 2 diabetes mellitus: implications for therapy. Treat Endocrinol. 2004;3(4):257-267.

17. Reitman ML, Schadt EE. Pharmacogenetics of metformin response: a step in the path toward personalized medicine. J Clin Invest. 2007;117(5):1226-1229.

18. Takane H, Shikata E, Otsubo K, Higuchi S, Ieiri I. Polymorphism in human organic cation transporters and metformin action. Pharmacogenomics. 2008;9(4):415-422.

19. Fowler M. Microvascular and macrovascular complication of diabetes. Diabetes Care. 2008;76(2):77-82.

20. Shikata E, Yamamoto R, Takane H, et al. Human organic cation transporter (OCT1 and OCT2) gene polymorphisms and therapeutic effects of metformin. J Hum Genet. 2007;52(2):117-122.

21. Shu Y, Sheardown SA, Brown C, et al. Effect of genetic variation in the organic cation transporter 1 (OCT1) on metformin action. J Clin Invest. 2007;117(5):1422-1431.

22. Becker ML, Visser LE, van Schaik RH, Hofman A, Uitterlinden AG, Stricker BH. Genetic variation in the multidrug and toxin extrusion 1 transporter protein influences the glucose lowering effect of metformin in patients with diabetes mellitus: a preliminary study. Diabetes. 2009;58(3):745-749.

23. Song IS, Shin HJ, Shim EJ, et al. Genetic variants of the organic cation transporter 2 influence the disposition of metformin. Clin Pharmacol Ther. 2008;84(5):559-562.

24. Kimura N, Masuda S, Tanihara Y, et al. Metformin is a superior substrate for renal organic cation transporter OCT2 rather than hepatic OCT1. Drug Metab Pharmacokinet. 2005;20(5):379-386.

25. Otsuka M, Matsumoto T, Morimoto R, Arioka S, Omote H, Moriyama Y. A human transporter protein that mediates the final excretion step for toxic organic cations. Proc Natl Acad Sci U S A. 2005;102(50): 17923-17928.

26. Moriyama Y, Hiasa M, Matsumoto T, Omote H. Multidrug and toxic compound extrusion (MATE)-type proteins as anchor transporters for the excretion of metabolic waste products and xenobiotics. Xenobiotica. 2008;38(7-8):1107-1118.

27. Tanihara Y, Masuda S, Sato T, Katsura T, Ogawa O, Inui K. Substrate specificity of MATE1 and MATE2-K, human multidrug and toxin extrusions/H(+)-organic cation antiporters. Biochem Pharmacol. 2007;74(2):359-371

28. Wang DS, Jonker JW, Kato Y, Kusuhara H, Schinkel AH, Sugiyama Y. Involvement of organic cation transporter 1 in hepatic and intestinal distribution of metformin. J Pharmacol Exp Ther. 2002;302(2):510-515.

29. Wang ZJ, Yin OQ, Tomlinson B, Chow MS. OCT2 polymorphisms and in-vivo renal functional consequence: studies with metformin and cimetidine. Pharmacogenet Genomics. 2008;18(7):637-645.

30. Tarasov AI, Nicolson TJ, Riveline JP, et al. A rare mutation in ABCC8/ SUR1 leading to altered ATP-sensitive $\mathrm{K}+$ channel activity and beta-cell glucose sensing is associated with type 2 diabetes in adults. Diabetes. 2008;57(6):1595-1604.

31. Koster JC, Remedi MS, Dao C, Nichols CG. ATP and sulfonylurea sensitivity of mutant ATP-sensitive K+ channels in neonatal diabetes: implications for pharmacogenomic therapy. Diabetes. 2005;54(9): 2645-2654.

32. Babenko AP, Polak M, Cave H, et al. Activating mutations in the ABCC 8 gene in neonatal diabetes mellitus. $N$ Engl J Med. 2006;355(5):456-466.

33. Gloyn AL, Pearson ER, Antcliff JF, et al. Activating mutations in the gene encoding the ATP-sensitive potassium-channel subunit Kir6.2 and permanent neonatal diabetes. $N$ Engl J Med. 2004;350(18): 1838-1849.

34. Tammaro P, Flanagan SE, Zadek B, et al. A Kir6.2 mutation causing severe functional effects in vitro produces neonatal diabetes without the expected neurological complications. Diabetologia. 2008;51(5): 802-810. 
35. Proks P, Shimomura K, Craig TJ, Girard CA, Ashcroft FM. Mechanism of action of a sulphonylurea receptor SUR1 mutation (F132L) that causes DEND syndrome. Hum Mol Genet. 2007;16(16):2011-2019.

36. Wagner VM, Kremke B, Hiort O, Flanagan SE, Pearson ER. Transition from insulin to sulfonylurea in a child with diabetes due to a mutation in KCNJ11 encoding Kir6.2-initial and long-term response to sulfonylurea therapy. Eur J Pediatr. 2009;168(3):359-361.

37. Sagen JV, Raeder H, Hathout E, et al. Permanent neonatal diabetes due to mutations in KCNJ11 encoding Kir6.2: patient characteristics and initial response to sulfonylurea therapy. Diabetes. 2004;53(10): 2713-2718.

38. Gloyn AL, Reimann F, Girard C, et al. Relapsing diabetes can result from moderately activating mutations in KCNJ11. Hum Mol Genet. 2005;14(7):925-934.

39. Pearson ER, Flechtner I, Njølstad PR, et al. switching from insulin to oral sulfonylurea's in patients with diabetes due to Kir6.2 mutations. N Engl J Med. 2006;355:467-477.

40. Feng Y, Guangyun M, Xiaowei R, et al. Ser1369Ala variant in sulfonylurea receptor gene $\mathrm{ABCC} 8$ is associated with antidiabetic efficacy of gliclazide in Chinese type 2 diabetic patients. Diabetes Care. 2008;31(10):1939-1944.

41. Becker ML, Visser LE, Trienekens PH, Hofman A, van Schaik RH, Stricker BH. Cytochrome P450 2C9 *2 and *3 polymorphisms and the dose and effect of sulfonylurea in type II diabetes mellitus. Clin Pharmacol Ther. 2008;83(2):288-292.

42. Pearson ER, Donnelly LA, Kimber C, et al. Variation in TCF7L2 influences therapeutic response to sulfonylureas: a GoDARTs study. Diabetes. 2007;56(8):2178-2182.

43. Aquilante CL. Pharmacogenetics of thiazolidinedione therapy. Pharmacogenomics. 2007;8(8):917-931.

44. Bohme M, Nitz I, Doring F, Klapper M. Analysis of the transcriptional regulation of the FABP2 promoter haplotypes by PPARgamma/ RXRalpha and Oct-1. Biochim Biophys Acta. 2008;1779(10):616-621.

45. Ludovico O, Pellegrini F, Di Paola R, et al. Heterogeneous effect of peroxisome proliferator-activated receptor gamma2 Ala12 variant on type 2 diabetes risk. Obesity (Silver Spring). 2007;15(5):1076-1081.

46. Bluher M, Lubben G, Paschke R. Analysis of the relationship between the Pro12Ala variant in the PPAR-gamma2 gene and the response rate to therapy with pioglitazone in patients with type 2 diabetes. Diabetes Care. 2003;26(3):825-831.
47. Deeb SS, Fajas L, Nemoto M, et al. A Pro12Ala substitution in PPARgamma2 associated with decreased receptor activity, lower body mass index and improved insulin sensitivity. Nat Genet. 1998;20(3): 284-287.

48. Masugi J, Tamori Y, Mori H, Koike T, Kasuga M. Inhibitory effect of a proline-to-alanine substitution at codon 12 of peroxisome proliferator-activated receptor-gamma 2 on thiazolidinedione-induced adipogenesis. Biochem Biophys Res Commun. 2000;268(1):178-182.

49. Kang ES, Park SY, Kim HJ, et al. Effects of Pro12Ala polymorphism of peroxisome proliferator-activated receptor gamma2 gene on rosiglitazone response in type 2 diabetes. Clin Pharmacol Ther. 2005; 78(2):202-208.

50. Snitker S, Watanabe RM, Ani I, et al. Changes in insulin sensitivity in response to troglitazone do not differ between subjects with and without the common, functional Pro12Ala peroxisome proliferatoractivated receptor-gamma2 gene variant: results from the Troglitazone in Prevention of Diabetes (TRIPOD) study. Diabetes Care. 2004;27(6): 1365-1368.

51. Florez JC, Jablonski KA, Sun MW, et al. Effects of the type 2 diabetesassociated PPARG P12A polymorphism on progression to diabetes and response to troglitazone. J Clin Endocrinol Metab. 2007;92(4): 1502-1509.

52. Kang ES, Park SY, Kim HJ, et al. The influence of adiponectin gene polymorphism on the rosiglitazone response in patients with type 2 diabetes. Diabetes Care. 2005;28(5):1139-1144.

53. Saad MF, Greco S, Osei K, et al; Ragaglitazar Dose-Ranging Study Group. Ragaglitazar improves glycemic control and lipid profile in type 2 diabetic subjects: a 12-week, double-blind, placebo-controlled dose-ranging study with an open pioglitazone arm. Diabetes Care. 2004;27(6):1324-1329.

54. Raskin P, Klaff L, McGill J, et al. Efficacy and safety of combination therapy: repaglinide plus metformin versus nateglinide plus metformin. Diabetes Care. 2003;26(7):2063-2068.

55. Zhang W, He YJ, Han CT, et al. Effect of SLCO1B1 genetic polymorphism on the pharmacokinetics of nateglinide. Br J Clin Pharmacol. 2006;62(5):567-572.

56. Kalliokoski A, Neuvonen M, Neuvonen PJ, Niemi M. Different effects of SLCO1B1 polymorphism on the pharmacokinetics and pharmacodynamics of repaglinide and nateglinide. J Clin Pharmacol. 2008;48(3):311-321.
Pharmacogenomics and Personalized Medicine

\section{Publish your work in this journal}

Pharmacogenomics and Personalized Medicine is an international, peer-reviewed, open access journal characterizing the influence of genotype on pharmacology leading to the development of personalized treatment programs and individualized drug selection for improved safety, efficacy and sustainability. This journal is indexed on the

\section{Dovepress}

American Chemical Society's Chemical Abstracts Service (CAS). The manuscript management system is completely online and includes a very quick and fair peer-review system, which is all easy to use. Visit http://www.dovepress.com/testimonials.php to read real quotes from published authors. 